\title{
Pion fluctuation in deep inelastic scattering
}

\author{
A. Bunyatyan ${ }^{1,2}$ a and B. Povh ${ }^{1} \mathrm{~b}$
}

${ }^{1}$ Max-Planck-Institut für Kernphysik, Postfach 103980, D-69029 Heidelberg, Germany

2 Yerevan Physics Institute, Alikhanian Brothers St.2, AM-375036 Yerevan, Armenia

\begin{abstract}
The forward neutron production in the ep collisions at $300 \mathrm{GeV}$ measured by $\mathrm{H} 1$ and ZEUS Collaborations at DESY has been used to estimate the total probability for proton fluctuation into $n \pi^{+}$ and $p \pi^{0}$. The probability found is on the order of $30 \%$. This number is compared with the numbers obtained for the probability of quark fluctuation into $\pi^{+}$from several alternative DIS processes (Gottfried sum rule, polarized structure function) and the axial-vector coupling constant, where the pion fluctuation is believed to play an important role.
\end{abstract}

PACS. 12.38.-t Quantum chromodynamics - 12.39.Jh Nonrelativistic quark model

\section{ก}

m Introduction

Measurements of the inclusive deep inelastic scattering (DIS) have allowed to extract important information on the partonic structure of the nucleon. Parton distributions have been determined and scaling violations have been tested to a high level of accuracy. The QCD parton model has been shown to be very reliable in the presence of the hard scale.

On the other hand, semi-inclusive reactions with electromagnetic probes are less explored. Through the study of new observables characterizing these processes we may ask more detailed questions about the hadronic structure. In semi-inclusive reactions the hadronic character of the nucleon becomes apparent, soft QCD becomes important. The most prominent effects in semi-inclusive DIS are the consequence of the strong correlation between the seaquark and the sea-antiquark in the relative state with the quantum number of the pion [1. We will refer to this correlation, as is the custom in the hadronic physics, as the pion fluctuation of the nucleon, $p \rightarrow N \pi$ and $p \rightarrow \Delta \pi$. A similar fluctuation of the proton to $K^{+} \Lambda$, which is less strong than the pion fluctuation because of higher excitation, is the source of the strange sea in the proton and explains the $s \bar{s}$ asymmetry in the neutrino DIS. Also the proton fluctuation to $D \Lambda_{c}$ may be the source of intrinsic charm in proton [2,3].

Two collaborations, H1 and ZEUS at HERA, have measured the production of forward neutrons in electronproton collisions at a center-of-mass energy of about 300 $\mathrm{GeV}$ 4,5,6,7,8,9. These events are successfully described within the models based on the pion exchange mechanism: the DIS takes place on the $\pi^{+}$fluctuation of the proton.

\footnotetext{
a e-mail: Armen.Bunyatyan@desy.de

b e-mail: B.Povh@mpi-hd.mpg.de
}

Neutrons carrying more than $50 \%$ of the proton initial energy produced in DIS on pions can be well distinguished from the neutrons produced in ordinary DIS on the proton by their energy and four-momentum distributions.

The forward neutron production with the aim to observe the pion fluctuation has already been studied previously in the high-energy $p p$-reaction at ISR in CERN 10. But the rescattering of proton on the virtual neutron is so strong that a reliable estimate of the probability of the $p \rightarrow \pi^{+} n$ fluctuation was not possible. On the other hand, the rescattering of the highly virtual photon $\left(Q^{2}>10 \mathrm{GeV}^{2}\right)$ on neutron is sufficiently small so that a reliable probability for the pion fluctuation can be deduced. Thus, the forward neutron production in DIS is probably the most direct way to determine the probability of the pion fluctuation quantitatively. The pion fluctuation is very likely responsible for the flavour asymmetry of the quark sea in the nucleon as observed in the violation of the Gottfried sum rule. Furthermore, the reduction of the amount of the angular momentum carried by the quark spins in the nucleon is probably also, to a great extent, a consequence of a pion fluctuation, as we will discuss later. These two phenomena as well as the value of the axial current coupling constant are directly related to the pion fluctuation of the quarks, $u \rightarrow d \pi^{+}, u \pi^{0}$ and $d \rightarrow u \pi^{-}, d \pi^{0}$. The pion Compton length is larger than the nucleon radius and the fluctuating pions overlap and interfere. They result in different Fock components of the nucleon with one, two and three pions.

Therefore we felt it interesting to estimate the probability of the pion fluctuation in the proton in DIS and compare it with the probability of the fluctuation of the quark. For the neutrons carrying large fraction of the initial proton energy the energy spectra agrees well with the pion exchange model predictions. At lower energies contributions from ordinary DIS are becoming important. The 
A. Bunyatyan and B. Povh: Pion fluctuation in deep inelastic scattering

H1 and ZEUS measurements were fitted by theoretical pion fluxes from which the pion probability in the nucleon can be deduced. Our present analysis investigates the uncertainty of this probability as a consequence of the different form of the neutron spectra as a function of the coupling constant and the formfactor. The contribution of the DIS on pion at low neutron energies was calculated by extrapolating the neutron spectrum from energies above $50 \%$ of the proton incoming energy to low energies and to full range of four-momentum transfer squared $(t)$ using three different pion fluxes which fitted the experimental data best.

\section{Estimate of the neutron flux from DIS on pion}

To evaluate the probability of pion fluctuation in DIS we use the measurements of the leading neutron production at HERA performed by $\mathrm{H} 1$ and ZEUS Collaborations. There the leading neutron production was studied in different processes: in the semi-inclusive DIS process $e p \rightarrow e n X$, in the photoproduction of $D^{*}$ and in the dijet production $4,5,6,7,8,9$. The kinematic range of leading neutron measurements are restricted by the geometrical acceptance of the forward neutron calorimeters to the range $x_{L} \gtrsim 0.2$ and $\theta_{n} \lesssim 0.8 \mathrm{mrad}$, where $x_{L}$ is the neutron energy relative to the initial proton energy and $\theta_{n}$ is the angle of the scattered neutron in the laboratory frame.

In our analysis we use only the measurements of the semi-inclusive channel $e p \rightarrow e n X$ which have the highest statistical significance and for which one expects the least distortion providing the measurement is done by sufficiently high $Q^{2}$. At low $Q^{2}$ the distortion can arise from the rescattering of the neutron on the extended hadronic photon the size of which increases with decreasing $Q^{2}$. The rescattering effects are expected to be small in the DIS regime, at sufficiently large photon virtualities $Q^{2}$, as is also confirmed by the measurements 7 .

Previous studies have shown that particle exchange models describe the leading neutron production data both in deep inelastic scattering at HERA and at hadroproduction [1] experiments. In these models the transition amplitude for $p \rightarrow n$ is dominated by $\pi^{+}$exchange [12. 13 14, 15. The interpretation of this process depends on the reference frame. In the rest frame of the proton DIS takes place from a pion emitted from the proton. In the infinite momentum frame (the rest frame of the photon) the photon emites the pion. Such ambiguities are resolved using light-front Fock methods 16. Let us first consider that the production of leading neutrons in DIS at large $x_{L}$ proceeds entirely via $p \rightarrow n+\pi^{+}$channel. Then the crosssection for photon-proton scattering to the final state $n X$ takes the form

$$
d \sigma^{\gamma^{*} p \rightarrow n X}=f_{\pi^{+} / p}\left(x_{L}, t\right) \cdot d \sigma^{\gamma^{*} \pi^{+} \rightarrow X},
$$

where $f_{\pi^{+} / p}\left(x_{L}, t\right)$ is the pion flux associated with the beam proton and $d \sigma^{\gamma^{*} \pi^{+} \rightarrow X}$ denotes the cross-section for the hard photon-pion interaction. The general form of pion flux is given by the expression

$$
f_{\pi^{+} / p}\left(x_{L}, t\right)=\frac{1}{2 \pi} \frac{g_{p \pi n}^{2}}{4 \pi}\left(1-x_{L}\right)^{1-2 \alpha(t)} \frac{-t}{\left(m_{\pi}^{2}-t\right)^{2}}|G(t)|^{2} .
$$

Here $m_{\pi}$ is the pion mass and $g_{p \pi n}^{2} / 4 \pi=13.7$ is the $p \pi n$ coupling constant, known from phenomenological analyses of low-energy data 17. $G$ is a formfactor which accounts for off-mass-shell corrections, normalized to be unity at the pion pole. For the light-cone approach $G$ becomes also dependent on $x_{L}$. The probability of pion fluctuation depends thus on $x_{L}$ and $t$. Our final result, called $<n \pi^{+} \mid p>^{2}$, corresponds to the probability integrated over $x_{L}$ and $t$ [18 19].

Several functional forms of pion flux can be found in the literature. These expressions differ in the assumptions for the formfactor $G$ and in the assumption of reggeization of the pion exchange (e.g. the value of $\alpha(t)$ ). In Fig 1 we show different predictions for the shape of the neutron energy distribution. The difference in the normalization should not affect our estimate as our analysis depends only on the ratios of the phase spaces. The extrapolation to the low energies and full $t$ range depends strongly on the choice of the flux.

We quote results using the pion fluxes of [13.14,15 which are also chosen by the experiments $4,5,6,7,8,9]$.

In ZEUS analysis 7 the fraction of leading neutron events was measured in the kinematic range $Q^{2}>4 \mathrm{GeV}^{2}$, and it was found to be $8.0 \pm 0.5 \%$ for $x_{L}>0.2$ or $5.8 \pm 0.3 \%$ for $x_{L}>0.49$. A similar result is obtained in the $\mathrm{H} 1$ measurements. The result is however published only in the PhD thesis [20, quoting the fraction of leading neutron events in DIS to be $\left(7.9_{-1.5}^{+2.0}\right) \%$ for $x_{L}>0.49,|t|<$ $0.5 \mathrm{GeV}^{2}$. In this analysis the inclination of proton beam with respect to the nominal value (beam tilt) was not properly taken into account. Considering the increase of geometrical acceptance for the neutrons by about $20 \%$ due to the observed proton beam tilt as was done for ref. 9 ] gives a corrected value of about $(6.3 \pm 1.5) \%$, which is in agreement with ZEUS results. Because of the larger errors of the value obtained by $\mathrm{H} 1$, only the ZEUS result will be used in further analysis.

Using the measured value of $(5.8 \pm 0.3) \%$ we estimate the full contribution of pion exchange to the DIS by extrapolating the measurement from the measured kinematical range to the full phase space. The result of this extrapolation is $(9.5 \pm 0.5) \%$ using the flux from [13], $(10.3 \pm 0.5) \%$ for flux [14] and $(14.6 \pm 0.7) \%$ for flux [15].

In this procedure we assumed that all events with leading neutrons in the kinematic range $x_{L}>0.49$ originate from pion exchange via $p \rightarrow n+\pi^{+}$channel. However, there are also other processes which can contribute. As was estimated in [13] in the kinematic range of the measurements, the leading neutron production due to $\rho$ and $a_{2}$ exchanges, to pomeron exchange, or to resonance decays, is about $10 \%$ of that due to pion exchange. The proton transition to $\Delta+\pi$ may also contribute to the production of leading neutrons. Measurements of $p \rightarrow n$ and $p \rightarrow \Delta^{++}$ 
reactions at Fermilab 21] indicate that contribution of $\Delta$ channel to forward neutron production is less than $6 \%$. This observation agrees with theoretical estimate of $\Delta+\pi$ contribution to be one third to two third of the $N+\pi$ probability [22, if we take into account all channels which can contribute to forward neutron production and the acceptance limitation due to larger angular spread and lower energies of neutrons produced from $\Delta$ decay.

Another effect which one has to take into account is the absorption or rescattering. It was pointed out by D'Alesio and Pirner 18 that also for the forward neutron production in $\gamma p \rightarrow n X$ reaction even for $Q^{2}>10 \mathrm{GeV}^{2}$ the rescattering of the virtual gamma is not negligible. For the $x_{L}>0.5$ the average reduction of the cross-section (e.g. increase of probability) is about $15 \%$.

Taking these effects into account the final values for the corrected fraction of the DIS on the pion compared to the total DIS cross-section is $(9.9 \pm 0.5) \%$ for the flux from [13, $(10.8 \pm 0.5) \%$ for the flux from [14] and $(15.2 \pm 0.7) \%$ for the flux from 15. The big discrepancy between the results calculated using fluxes from [13 14] and flux from [15] is the consequence of the fact that in the flux [15] a constant formfactor was assumed. The discrepancy of $30 \%$ between the fluxes comes from the contribution to the extrapolated cross-section from the $x_{L}<0.49$ region.

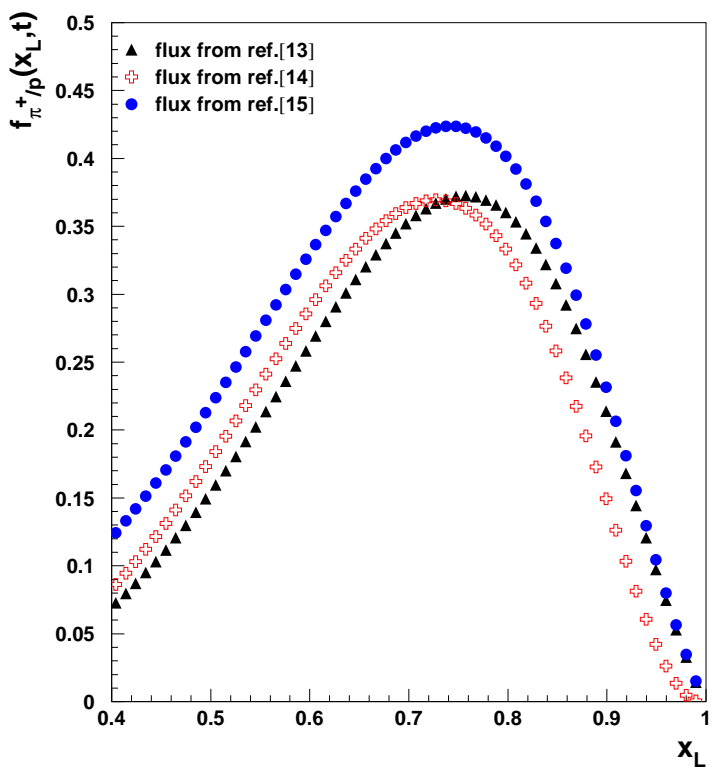

Fig. 1. The pion flux as function of $x_{L}$ for different shapes of the formfactor integrated over $t$ in the range determined by the angular acceptance of the forward neutron calorimeter.
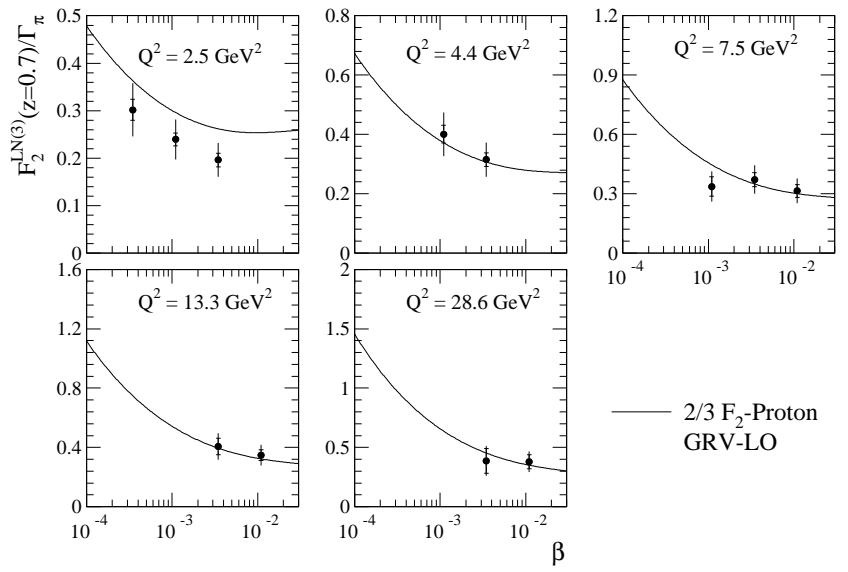

Fig. 2. The H1 measurement of $F_{2}^{L N} / \Gamma_{\pi}$ plotted as a function of $\beta \equiv x_{\pi}$ for fixed values of $Q^{2}$, where $\Gamma_{\pi}$ is the integrated pion flux factor. $F_{2}^{L N} / \Gamma_{\pi}$ can be interpreted as being the pion structure function $F_{2}^{\pi}$. The data are compared to the GRV-LO [23. parameterization of $F_{2}$ of the proton scaled by $2 / 3$.

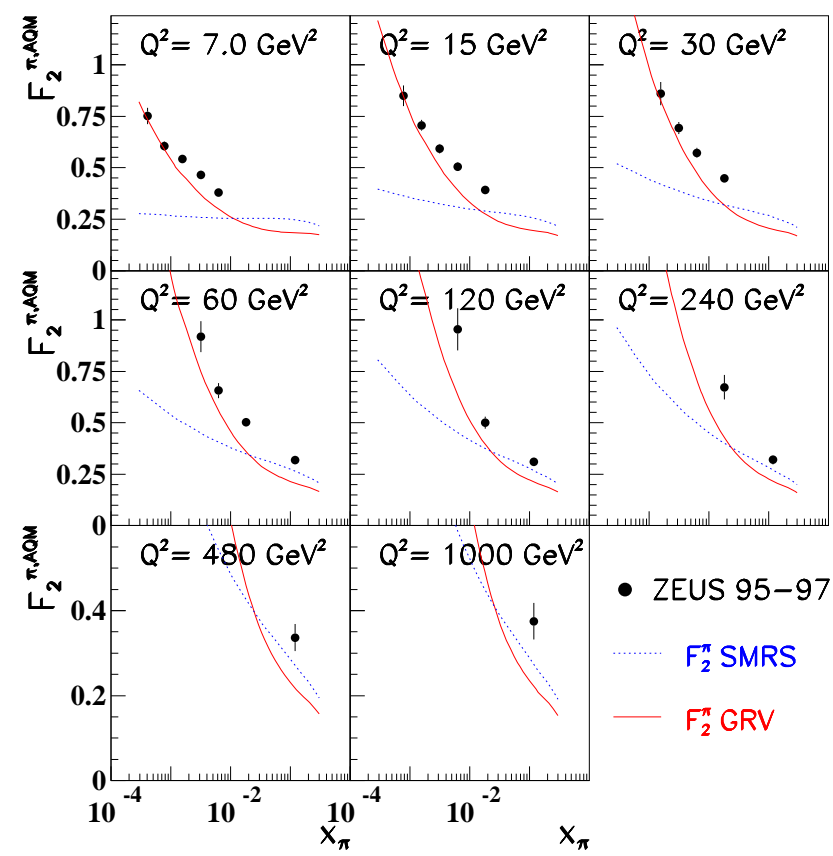

Fig. 3. The ZEUS measurement of $F_{2}^{\pi}$ in the range $0.64<$ $x_{L}<0.82$ as a function of $x_{\pi}$ for fixed $Q^{2}$. The data are compared to the GRV 25] and SMRS [26] parameterizations of $F_{2}^{\pi}$.

\section{Probability of pion fluctuation observed in DIS}

To estimate the full probability of the $p \rightarrow \pi^{+} n$ fluctuation, one needs to know the relative cross-section of DIS on pion compared to DIS on proton. For $x_{L} \sim 1$ the pion is close to mass shell and one expects the ratio of pion cross-section to total cross-section to be $2 / 3$. The ratio of 
$F_{2}^{\pi}$, measured in $\mathrm{H} 1$ in the range $0.7<x_{L}<0.9$, to $F_{2}$ is consistent with the ratio of $2 / 3$ [4]24] (Fig 221). In this measurement the flux from [13] was used for normalization. In the ZEUS analysis [5] two different normalizations of flux factor were considered. The first method gives ratio of $F_{2}^{\pi}$ to $F_{2}$ equal to 0.361 . Here the normalization was done using the effective flux which was taken from hadronic interactions [15]. This result contradicts the expectation that for $x_{L}=1$ the ratio is $2 / 3$. The second method used by ZEUS assumes the ratio of $\gamma^{*} \pi$ to $\gamma^{*} p$ cross-sections to be $2 / 3$ at $x_{L}=1$. This result is in good agreement with the GRV parameterization [25] of $F_{2}^{\pi}$ (Fig[3). The difference in the center-of-mass energies of $\gamma^{*} \pi$ and $\gamma^{*} p$ is also taken into account as

$$
\frac{\sigma^{\gamma^{*} \pi}\left(s\left(1-x_{L}\right)\right)}{\sigma^{\gamma^{*} p}(s)}=\frac{2}{3}\left(1-x_{L}\right)^{\alpha_{\mathbb{P}}(0)-1}=0.6 .
$$

The probability of the $\pi^{+}$fluctuation of proton is then $(16.5 \pm 0.8) \%$ for the flux from [13, $(18.0 \pm 0.9) \%$ for [14] and $(25.3 \pm 1.2) \%$ for 15 . Using the isospin symmetry the fluctuation of $p \rightarrow p+\pi^{0}$ is half of these numbers. The full probability of the pion fluctuation of the proton is then $(27.0 \pm 1.2) \%$ for the flux from [13], $(24.8 \pm 1.3) \%$ for [14] and $(38.0 \pm 1.8) \%$ for [15].

We note that the errors are entirely experimental errors. The systematic errors of our evaluation are reflected in the spread of the numbers for probabilities assuming different forms of pion fluxes.

\section{Discussion}

The pion fluctuation of the nucleon in the quark model has been so far investigated in inclusive deep inelastic scattering: Gottfried sum rule, spin structure functions. From these experiments the parameter which defines the probablity of quark fluctuation into pion, $a=\left\langle d \pi^{+} \mid u\right\rangle^{2}$, can be extracted.

The importance of the pion fluctuation in DIS was first realized in the violation of Gottfried sum rule (GSR) [27. 28. The GSR expressed in parton distribution functions separated in valence quarks $\left(u_{v}, d_{v}\right)$ and sea quarks $(\bar{u}, \bar{d})$ reads

$$
\begin{aligned}
I_{G}\left(0,1 ; Q^{2}\right)= & \frac{1}{3} \int_{0}^{1} d x\left[u_{v}\left(x, Q^{2}\right)-d_{v}\left(x, Q^{2}\right)\right] \\
& -\frac{2}{3} \int_{0}^{1} d x\left[\bar{u}\left(x, Q^{2}\right)-\bar{d}\left(x, Q^{2}\right)\right] .
\end{aligned}
$$

For the symmetric sea

$$
I_{G}\left(0,1, Q^{2}\right)=\frac{1}{3}
$$

is obtained by noting that the first term in (4) becomes simply the difference between the number of up and down valence quarks in a proton, times $1 / 3$.
The second integral of (4) is straitforward to evaluate, if one assumes that the entire sea asymmetry comes from the pion fluctuation

$$
\int_{0}^{1} d x\left[\bar{u}\left(x, Q^{2}\right)-\bar{d}\left(x, Q^{2}\right)\right]=a\left(Q^{2}\right) .
$$

and

$$
I_{G}=\frac{1}{3}-\frac{2}{3} a
$$

The first published results of the GSR by the New Muon Collaboration [27] quotes

$$
I_{G}\left(0,1 ; Q_{0}^{2}=4 \mathrm{GeV}^{2}\right)=0.240 \pm 0.016
$$

This number has been afterwards corrected for the shadowing in the deuteron measurement 29] resulting to be $I_{G}=0.216 \pm 0.024$ (exp.) \pm 0.009 (shad.). From this number using (7) the probability for the pion fluctuation $a=$ $0.18 \pm 0.05$. The fluctuation of the nucleon into $\Delta+\pi$ also contributes to the correction to GSR with the same sign as $N \pi$ fluctuation.

The model used by Eichten et al. 28 to calculate the violation of the GSR we can consider as the lowest order of the flavour $\mathrm{SU}(2)$-chiral model of the nucleon. Within this model Pirner [30] gives an expression for the integral of the spin structure function as a function of $a$ as well as the expression for the axial-vector coupling constant $g_{A}$.

The integral of the spin structure function $I_{p}$ can be written as

$$
I_{p}=\frac{5}{18}(1-2 a)
$$

$I_{p}$ was measured by EMC 31] as $I_{p}=0.126 \pm 0.010$ (stat.) \pm 0.015 (syst.), from which the number $a$ can be obtained as $a=0.273 \pm 0.030$.

The next best experimentally measured spin structure function is for the deuterium. It was measured by E143 32 as $I_{d}=0.042 \pm 0.005$. The number $a$ is obtained from

$$
I_{D}=\frac{5}{18}(1-3 a)
$$

as $0.283 \pm 0.040$.

We further comment on an alternative estimate of $I_{P}$ and $I_{D}$. In order to avoid the explicite writing-down of the baryon wave functions one assumes the validity of the flavour SU(3) for the hyperon octet. In the SU(3) model the semi-leptonic weak decays can be well fitted by two parameters [33], the irreducible matrix elements of the $\mathrm{SU}(3)$ representation, $\mathrm{F}$ and $\mathrm{D}$. The flavour SU(3) model is liked by theorists as it avoids writing explicitly the wave functions, but assures their proper antisymmetrisation. It may be of some disadvantage when applied to the spin structure functions. The axial-vector coupling constant expressed in the model is $g_{A}=F+D$, and is fitted to the experimentally measured value $g_{A}=1.267 \pm 0.003$. In the $\mathrm{SU}(3)$ model the spin structure function depends also on the ratio $\mathrm{F} / \mathrm{D}$. This ratio is determined by optimizing the fit of the semi-leptonic decays of the hyperons, all of them having $\Delta S=1$, and takes into account possible 
kaon fluctuation of the proton. The $\mathrm{SU}(3)$ prediction for the integrals $I_{P}$ and $I_{D}$ using the best known values of $\mathrm{F}$ and D from Cabibbo et al. 33 . corresponds in the $\mathrm{SU}(2)$ chiral model to $a \approx 0.19$, supporting our assumption that the main contribution to the spin reduction comes from the pion fluctuation.

The axial current coupling constant may give an independent information on the probability of the pion fluctuation in the proton ground state. It was early realized that the experimental value of the axial-vector current coupling constant of the weak decays $g_{A}$ can be reproduced if the pion coupling to the quarks is also taken into account in the axial-vector current (partially conserved axial-vector current) 34,35. However, the estimate of the pion contribution is srongly model dependent. In the $\mathrm{SU}(2)$ chiral model 30]

$$
g_{A}=(1-a) \frac{5}{3}
$$

From the measured value of $g_{A}=1.267 \pm 0.003$ and using eq11 we obtain $a=0.240 \pm 0.002$.

We want to compare our evaluation of the probability of pion fluctuation in the proton $\left(P_{N \pi}\right)$ with values of $a$ obtained from previously discussed inclusive processes. In our evaluation we used data which explicitly require leading neutrons in the final state. The relation between $P_{N \pi}$ and $a$ is not obvious. $a$ is the probability of pion fluctuation on the quark level, and $P_{N \pi}$ is the probablity of pion fluctuation on the nucleon level. To connect these quantities one would need the knowledge of the microscopic wave function of the nucleon.

In Fig 4 our values of the probability of the proton fluctuation into $n+\pi^{+}$(which is $2 / 3 P_{N \pi}$ ) are shown together with the values of $a$, the $u$-quark fluctuation in $d+\pi^{+}$, from different processes. In view of the very crude approximation of pion fluctuation being the ground state and ignoring the $Q^{2}$ dependences, the agreement between the different values should be considered as reasonably good. The observed similarity of the values of $a$ and $P_{N \pi}$ demonstrated clearly that $a$ is not an additive quantity 36.19, the pion cloud from different quarks overlap and interfere. It is also another demonstration of the importance of soft interactions in semi-inclusive processes.

\section{Acknowledgments}

We wish to thank B. Kopeliovich, H.J. Pirner and W. Weise for valuable discussions and useful remarks.

\section{References}

1. G. Altarelli, N. Cabibbo, L. Maiani and R. Petronzio, Nucl. Phys. B 69 (1974) 531.

2. S. J. Brodsky, C. Peterson and N. Sakai, Phys. Rev. D 23 (1981) 2745.

3. M. Franz, M. V. Polyakov and K. Goeke, Phys. Rev. D 62 (2000) 074024 [hep-ph//0002240].

4. C. Adloff et al. [H1 Collaboration], Eur. Phys. J. C 6 (1999) 587 hep-ex/9811013.

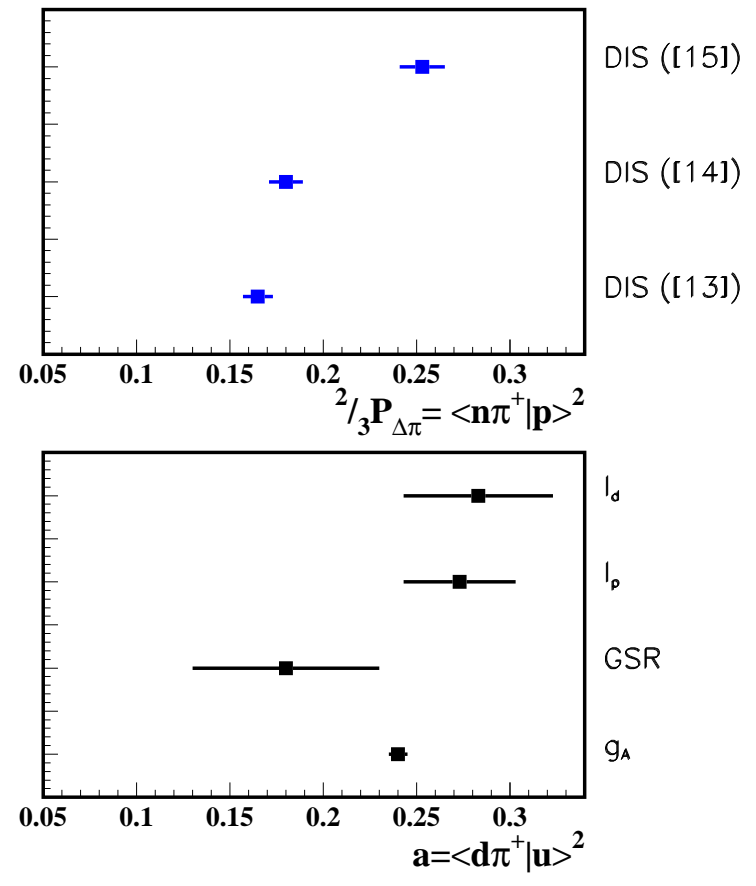

Fig. 4. Comparison of the values of the $p \rightarrow n \pi^{+}$fluctuation from DIS with $a$ from different experiments. DIS ([13, 14, 15]) are our values of $2 / 3 P_{N \pi}$ probability using pion fluxes from [13, 14] and 15], respectively; $I_{d}$ and $I_{p}$ are values of $a$ ( $u \rightarrow d+\pi^{+}$fluctuation) obtained from integrals of polarized structure functions on deuteron and proton; GSR is the value of $a$ obtained from the Gottfried sum rule; $g_{A}$ is the value of $a$ from the axial-vector coupling.

5. S. Chekanov et al. [ZEUS Collaboration], Nucl. Phys. B 637 (2002) 3 hep-ex/0205076.

6. S. Chekanov et al. [ZEUS Collaboration], Phys. Lett. B 610 (2005) 199 hep-ex/0404002.

7. S. Chekanov et al. [ZEUS Collaboration], Phys. Lett. B 590 (2004) 143 hep-ex/0401017.

8. S. Chekanov et al. [ZEUS Collaboration], Nucl. Phys. B 596 (2001) 3 hep-ex/0010019.

9. A. Aktas et al. [H1 Collaboration], Eur. Phys. J. C 41 (2005) 273 hep-ex/0501074.

10. J. Engler et al., Nucl. Phys. B 84 (1975) 70; W. Flauger and F. Mönnig, Nucl. Phys. B 109 (1976) 347.

11. B. Robinson et al. Phys. Rev. Lett. 34 (1975) 1475.

12. J.D. Sullivan, Phys. Rev. D 5 (1972) 1732.

13. B. Kopeliovich, B. Povh and I. Potashnikova, Z. Phys. C 73 (1996) 125 hep-ph/9601291.

14. H. Holtmann et al., Phys. Lett. B 338 (1994) 363.

15. M. Bishari Phys. Lett. B 38 (1972) 510.

16. S. J. Brodsky, Nucl. Phys. Proc. Suppl.90 (2000) 3 hep-ph/0009229.

17. R.G.E. Timmermans, Th.A. Rijken and J.J. de Swart, Phys. Rev. Lett. 67 (1991) 1074.

18. U. D'Alesio and H.J. Pirner, Eur. Phys. J. A 7 (2000) 109 hep-ph/9806321.

19. W. Koepf, L. Frankfurt and M. Strikman, Phys. Rev. D 53 (1996) 2586 hep-ph/9507218. 
20. F. Metlica, PhD thesis, University of Heidelberg, 1998.

21. J. Erwin el al., Phys. Rev. Lett. 35 (1975) 980; P.D. Higgins el al., Phys. Rev. D 19 (1979) 731; S.J. Barish el al., Phys. Rev. D 12 (1975) 1260; F.T. Dao el al., Phys. Rev. Lett. 30 (1973) 34.

22. A. W. Thomas and C. Boros, Eur. Phys. J. C 9 (1999) 267 hep-ph/9812264.

23. M. Glück, E. Reya and A. Vogt, Z. Phys. C 53 (1992) 127.

24. T. Nunneman, PhD thesis, University of Heidelberg, 1998.

25. M. Glück, E. Reya and A. Vogt, Z. Phys. C 53 (1992) 651.

26. P.J. Sutton, et al. Phys. Rev. D 45 (1992) 2349.

27. P. Amaudruz et al. [NMC Collaboration], Phys. Rev. Lett. 66 (1991) 21;

M. Arneodo et al. [NMC Collaboration], Phys. Rev. D 50 (1994) 1.

28. E.J.Eichten, I.Hinchliffe and C.Quigg, Phys. Rev. D 45 (1992) 2269.

29. A. Brüll, Habilitation thesis, University of Heidelberg 1995.

30. H.J. Pirner, Prog. Part. Nucl. Phys.36 (1996) 19.

31. J. Ashman et al. [EMC Collaboration], Nucl. Phys. B 328 (1989) 1.

32. K. Abe et al., [E143 Collaboration], Phys. Rev. Lett. 75 (1995) 25.

33. N. Cabibbo, E.C. Swallow and R. Winston, Ann. Rev. Nucl. Part. Sci. 53 (2003) 39 hep-ph/0307298.

34. S. L. Adler, Phys. Rev. Lett. 14 (1965) 1051.

35. W. I. Weisberger, Phys. Rev. Lett. 14 (1965) 1047.

36. N.N Nikolaev al, Phys. Rev. D 60 (1999) 014004 hep-ph/9812266. 